\title{
Figure of Merit Enhancement of Surface Plasmon Resonance Biosensor Using Ga-Doped Zinc Oxide in Near Infrared Range
}

\author{
Sarika PAL ${ }^{1}$, Alka VERMA $^{2}$, Y. K. PRAJAPATI ${ }^{3}$, and J. P. SAINI ${ }^{4}$ \\ ${ }^{1}$ Department of ECE, National Institute of Technology Uttarakhand, Srinagar, Garhwal 246174, India \\ ${ }^{2}$ Department of ECE, Institute of Engineering and Rural Technology, Allahabad, Prayagraj, Uttar Pradesh 211002, \\ India \\ ${ }^{3}$ Department of ECE, Motilal Nehru National Institute of Technology Allahabad, Prayagraj, Uttar Pradesh 211004, \\ India \\ ${ }^{4}$ Department of ECE, Netaji Subhas University of Technology (NSUT), New Delhi 110078, India \\ *Corresponding author: Alka VERMA_E-mail: alkapra25@gmail.com
}

\begin{abstract}
This work presents a surface plasmon resonance biosensor for the figure of merit enhancement by using Ga-doped zinc oxide (GZO), i.e., nanostructured transparent conducting oxide as plasmonic material in place of metal at the telecommunication wavelength. Two-dimentional graphene is used here as a biorecognition element (BRE) layer for stable and robust adsorption of biomolecules. This is possible due to stronger van der Waals forces between graphene's hexagonal cells and carbon-like ring arrangement present in biomolecules. The proposed sensor shows improved biosensing due to fascinating electronic, optical, physical, and chemical properties of graphene. This work analyses the sensitivity, detection accuracy, and figure of merit for the GZO/graphene SPR sensor on using the dielectric layer in between the prism and GZO. The highest figure of merit of $366.7 \mathrm{RIU}^{-1}$ is achieved for the proposed SPR biosensor on using the nanostructured GZO at the $3000 \mathrm{~nm}$ dielectric thickness. The proposed SPR biosensor can be used practically for sensing of larger size biomolecules with due availability of advanced techniques for the fabrication of the nanostructured GZO and graphene.
\end{abstract}

Keywords: SPR; transparent conducting oxide; Ga-doped zinc oxide; sensitivity $(S)$; detection accuracy; figure of merit

Citation: Sarika PAL, Alka VERMA, Y. K. PRAJAPATI, and J. P. SAINI, "Figure of Merit Enhancement of Surface Plasmon Resonance Biosensor Using Ga-Doped Zinc Oxide in Near Infrared Range," Photonic Sensors, 2020, 10(4): 340-352.

\section{Introduction}

Since last few decades, optical sensors, especially surface plasmon resonance (SPR) sensors, have gained much popularity for gas sensing, chemical sensing, and biosensing applications [1-3]. The SPR sensors are used to detect a change in refractive index (RI) of the sensing medium, which is very sensitive towards the exponential decaying fields of the surface plasmon wave (SPW) at the metal and dielectric interface. The SPR sensors are the most commonly used sensors for their high sensitivity, accuracy, reliability, label-free, and fast detection procedures [3]. Based on the light 
coupling mechanism, the SPR sensors are categorized into different types like grating coupled, prism coupled, waveguide coupled, and fiber optic SPR sensors. Prism coupled SPR sensors are preferable as they can be easily realized while the fiber-optic SPR sensor suffers from the efficient launching of light inside the fiber [4]. There are two types of configuration namely Kretschmann and Otto configurations for prism coupled SPR sensors. Kretschmann configuration-based prism coupled SPR sensors, in which the plasmonic thin metal film is deposited onto flat face of the prism, are frequently investigated due to their possible realization [4]. For this geometry, when TM polarized light is incident from one side of the prism at an angle greater than the critical angle, it penetrates through the metal prism, and an evanescent wave is generated. The evanescent wave excites surface plasmon wave (SPW) at the metal-dielectric interface when the wave vector of the evanescent wave is matched with the surface plasmon wave $\left[k_{\mathrm{ev}}=\operatorname{Real}\left\{k_{\mathrm{spw}}\right\}\right]$ [5]. The spatial extension of surface plasmons (SPs) in the sensing medium is characterized by two parameters: (1) field penetration depth in the transverse direction and (2) propagation length of SPs in the lateral direction. Propagation length (PL) is basically a distance covered by SPs in the direction of propagation which decays exponentially by the factor of e due to losses within the metal [4]. Penetration depth (PD) measures the interaction length of SPs in the transverse direction over which SPs are sensitive to detect refractive index change of the sensing medium due to binding of biomolecules on the sensing surface. On measuring reflectance through the photodetector, SPR condition causes a dip in reflectance due to the complete transfer of energy from an evanescent wave to SPW. The resonance angle responsible for the surface plasmon resonance condition depends on the refractive index and thicknesses of the metal film and dielectric medium used.
The conventional SPR sensors commonly use noble metals such as gold and silver for surface plasmon polaritons (SPPs) generation in the visible region [5]. But these metals suffer from large optical losses in the near-infrared (NIR) region limiting SPR sensor performance [6]. NIR is the most suitable optical range for biosensing applications as bio-samples remain transparent and are not affected by photo-damage in this range [7]. So, low-loss plasmonic materials like nanostructured transparent conducting oxide (TCO) i.e., Ga-doped zinc oxide (GZO) can be tried as an alternative in place of the conventional metal in the NIR range $[8,9]$. West et $a l$. verified that in the infrared region TCO exhibited four times smaller losses than silver [6]. TCO gives smaller losses and is more tunable for SPs generation in comparison with metals in the infrared range. This is due to higher carrier concentration of TCOs (i.e., $10^{20} \mathrm{~cm}^{-3}$ ) than those of noble metals in the infrared range. TCOs below this concentration behave as the dielectric and cannot be used for SPs generation. Optimized and careful control of doping concentration, doping type, deposition temperature, and pressure lead to a proper fabrication of TCOs for their application in plasmonic devices. TCOs are semiconductor-based design having smaller optical losses, tunable, and compatible to standard nanofabrication processes [10]. Kim et al. experimentally verified the plasmonic behavior of GZO for the SPR sensor in the NIR region [9]. But broader SPR curves obtained resulted in poor detection accuracy (DA) and figure of merit (FOM). The use of the dielectric layer between the prism and TCO may offer a solution to improve the DA and FOM of the surface plasmon resonance sensor for the accurate detection of biomolecules [11]. This gives a longer penetration depth, a higher electromagnetic field at the metal/dielectric interface, and sharper SPR curves in comparison with the conventional SPR sensors. The longer penetration depth of the evanescent field at the sensing surface in the NIR range gives the possibility for the 
detection of larger size biomolecules [12]. Sarid introduced an idea to improve the DA and FOM of the SPR sensor [13]. As per his findings, if the thin metal layer is kept between two dielectrics of similar RIs, i.e., $\varepsilon_{1}$ and $\varepsilon_{2}$, then the DA and FOM of the SPR sensor can be improved. However, the performance of the SPR sensor for biosensing also depends on methods of immobilization of biomolecules and their attachment on the sensing surface [2]. Two-dimentional (2D) graphene, a one-atom-thick sheet of carbon atoms, was efficiently used as a bio-recognition element (BRE) layer in SPR biosensing applications which has also been proved experimentally [14]. A sensitivity enhancement of $25 \%$ was observed using 10 layers of graphene [14]. Fascinating electrical, optical, physical, and chemical properties of graphene have attracted research for its application in SPR biochemical and biosensing [14-17]. Commendable research growth on graphene based SPR sensors have been possible, not only due to their novel and unique properties but also due to their fabrication possibilities. Tan et al. experimentally verified the plasmonic application of graphene after fabricating it by using the chemical vapour deposition technique [18]. They insisted on the use of graphene for sensing application where high sensitivity and high resolution was required. Zhao et al. proposed a highly sensitive SPR biochemical sensor which utilized a heterostructure of transition metal dichalcogenides (TMDC) and graphene [19]. They achieved an angular sensitivity of $315.5 \%$ RIU for $36 \mathrm{~nm}$ aluminium thin film and 7 layers of $\mathrm{WS}_{2}$. Recently, Chen et al. compared sensitivities of the graphene based SPR biosensor for $\mathrm{Au}, \mathrm{Cu}$, and $\mathrm{Ag}$ metals in the visible region [20]. They obtained the highest sensitivity of $300.26^{\circ}$ / RIU with $\mathrm{Ag}$ which was $119 \%$ and $200 \%$ higher than those by using $\mathrm{Au}\left(137.02^{\circ} / \mathrm{RIU}\right)$ and $\mathrm{Cu}$ $(136.24 \%$ RIU) for monolayer graphene, respectively. Very recently, Liu et al. proposed a highly sensitive SPR biosensor based on graphene and barium titanate layers for the $\mathrm{Ag}-\mathrm{Au}$ bimetallic configuration in the visible regime [21]. They achieved the highest angular sensitivity (294\%/RIU) and FOM (42.13 $\mathrm{RIU}^{-1}$ ) after optimizing proposed configuration. Very recently, Hossain et al. proposed a Kretchmann configuration based SPR biosensor for formalin detection which utilized the graphene/ $\mathrm{MoS}_{2}$ hybrid structure along with $\mathrm{TiO}_{2}$ and $\mathrm{SiO}_{2}$ nanoparticles [22]. They achieved the maximum sensitivity of $85.375 \%$ for bilayers of $\mathrm{TiO}_{2}$ and $\mathrm{SiO}_{2}$. This showed that till today, a large number of researchers investigated the highly sensitive graphene based SPR biosensor, where metals had been used for SPs generation [18-22]. Very few of them focused on enhancing other parameters like DA and FOM for the accurate detection of biomolecules [21]. FOM is product of sensitivity and DA, and its higher value provides us an optimized performance of SPR biosensor and accuracy. Until today, researchers have not tried an FOM enhancement for the graphene based SPR biosensor where TCOs are used for SPs generation in the infrared range. So, here we propose a graphene based SPR biosensor for the FOM enhancement which utilizes nanostructured GZO i.e., TCO for SPs generation instead of using metals rich in electrons.

In this work, we propose an SPR sensor in which GZO based nanostructured TCO is used as the plasmonic material in place of conventional metals and graphene as the BRE for biosensing at the telecommunication wavelength i.e., $1550 \mathrm{~nm}$. Performance of the SPR biosensor is analyzed for plasmonic nanostructured TCO i.e., GZO used for SPs generation. We analyze the performance of the SPR biosensor for two sensor designs: (1) prism/GZO/graphene/sensing medium and (2) $\mathrm{prism} /$ dielectric/GZO/graphene/sensing medium. The performance of the proposed SPR sensor (i.e., prism/dielectric/GZO/graphene/sensing medium) is analyzed to get improved DA and FOM for sensing of large-size biomolecules. The performance of the proposed sensor is also compared with that of the 
conventional SPR sensor. The complete work is organized as follows: Section 2 explains the proposed sensor design and theoretical modeling, Section 3 explains results and analysis, and Section 4 concludes the work presented.

\section{Proposed sensor modeling}

The related section describes the proposed sensor design and transfer matrix method for reflectance calculation through $N$-Layer modeling for the proposed SPR biosensor.

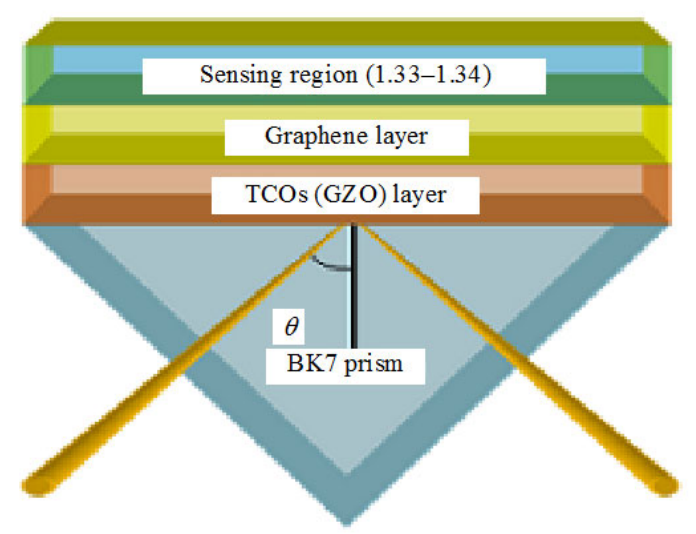

\subsection{Proposed sensor design}

The schematic diagram for the Kretschmann configuration based proposed SPR biosensor is shown in Figs. 1(a) and 1(b).

The proposed sensor design consists of the BK-7 prism, the layer-wise arrangement of the dielectric layer, GZO transparent conducting oxide for SPPs generation, graphene layer, and sensing medium. The SPR sensor design shown in Fig. 1(a) is for the sensor design prism/GZO/graphene/sensing medium.

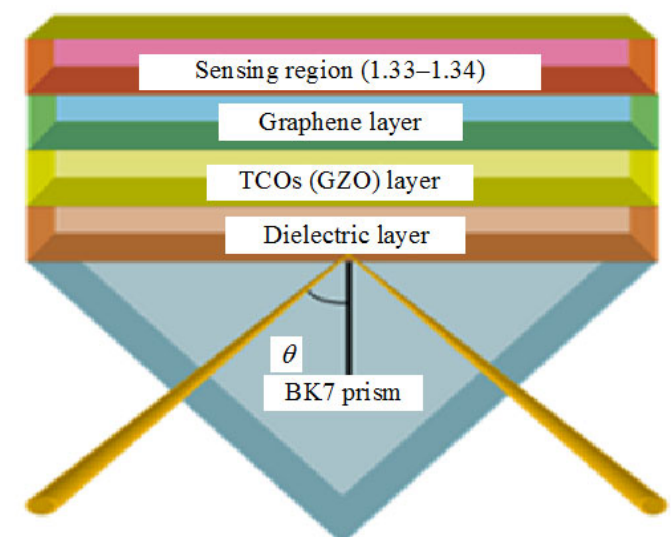

Fig. 1 SPR sensor design: (a) prism/GZO/graphene/sensing medium and (b) prism/dielectric/GZO/graphene/sensing medium.

Figure 1(b) shows a schematic diagram for the proposed sensor design i.e., prism/dielectric/GZO/graphene/sensing medium. An angular interrogation method is used to analyze the sensor's performance analytically. The operating wavelength of $1550 \mathrm{~nm}$ is chosen in this NIR range, GZO shows a plasmonic response, and biosensing can be performed efficiently [7]. The RI of the BK-7 prism at $1550 \mathrm{~nm}$ operating wavelength is 1.501 . GZO is used here as a plasmonic material to show metals like behavior in the NIR range. GZO, which possesses a smaller loss, is used here for SPPs generation instead of using noble metals like gold and silver which show much lossy behavior in the NIR range. The dielectric constant for GZO is calculated by using the Drude-Lorentz oscillator model and expressed in (1):

$$
\varepsilon(\omega)=\varepsilon_{\infty}+\frac{f_{1} \omega_{l}^{2}}{\omega_{l}^{2}-\omega^{2}-\mathrm{i} \omega \Gamma_{l}}-\frac{\omega_{p}^{2}}{\omega^{2}+\mathrm{i} \omega \Gamma_{p}}
$$

where $\omega, \omega_{l}, \omega_{p}, \varepsilon_{\infty}, f_{1}, \Gamma_{l}$, and $\Gamma_{p}$ are the light frequency, central frequency, unscreened plasma frequency, background permittivity, the strength of the Lorentz oscillator, damping and carrier relaxation rates, respectively. The value of Drude-Lorentz oscillator's parameter for GZO is shown in Table 1.

Table 1 Drude-Lorentz oscillator's parameter for GZO.

\begin{tabular}{ccccccc}
\hline TCO parameters & $\varepsilon_{\infty}$ & $\omega_{p}(\mathrm{eV})$ & $\Gamma_{p}(\mathrm{eV})$ & $f_{1}$ & $\omega_{l}(\mathrm{eV})$ & $\Gamma_{l}(\mathrm{eV})$ \\
\hline $\mathrm{GZO}$ & 2.475 & 1.927 & 0.117 & 0.866 & 4.850 & 0.029 \\
\hline
\end{tabular}

The GZO thickness chosen for the GZO/Graphene SPR biosensor [SPR sensor design: Fig. 1(b)] is $50 \mathrm{~nm}$, which is the same as the thickness chosen for the gold layer in the conventional SPR biosensor. The same thickness of gold as well as GZO, i.e., $50 \mathrm{~nm}$, is taken for the conventional SPR as well as the GZO/graphene SPR 
to compare the performances of both sensors in replacement of the gold layer by GZO i.e., TCO for SPs generation. But the larger full-width at half maximum (FWHM) of $19.99^{\circ}$ for the GZO/Graphene SPR gives a poor DA which may be improved by using the dielectric layer between the prism and GZO i.e., our proposed biosensor design (prism/dielectric/GZO/graphene/sensing medium). Finally, we optimize GZO and dielectric thicknesses for the proposed sensor design to improve its

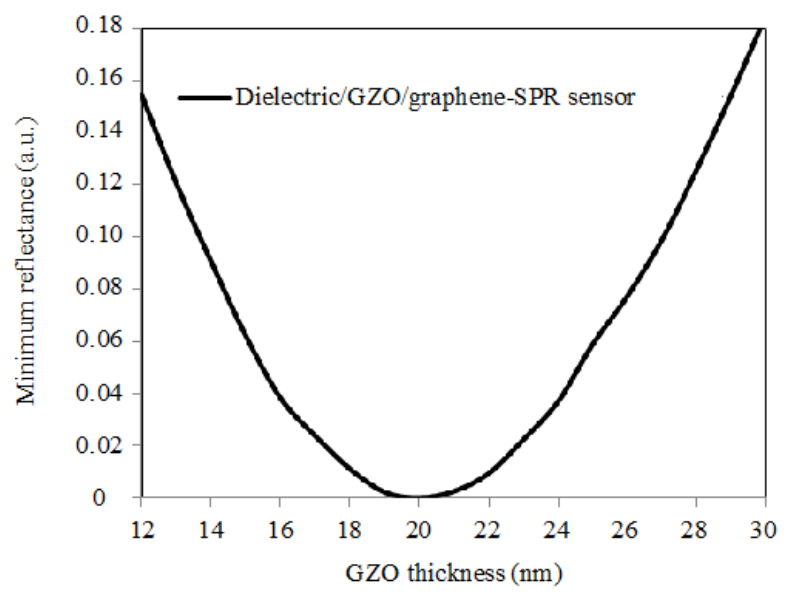

(a) performance mainly in terms of DA and FOM. To get an optimized thickness of the GZO and dielectric layers, we plot Figs.2(a) and 2(b) from the results obtained through SPR curves. Figure 2(a) shows the minimum reflectance obtained for different thicknesses of GZO on keeping dielectric thickness fixed at $2000 \mathrm{~nm}$. The minimum reflectance $\left(R_{\text {min. }}\right)$ is calculated for GZO thicknesses variation from $12 \mathrm{~nm}$ to $30 \mathrm{~nm}$. The minimum reflectance $\left(R_{\text {min. }}\right)$ of 0 a.u. is obtained at $20 \mathrm{~nm}$ thickness of GZO.

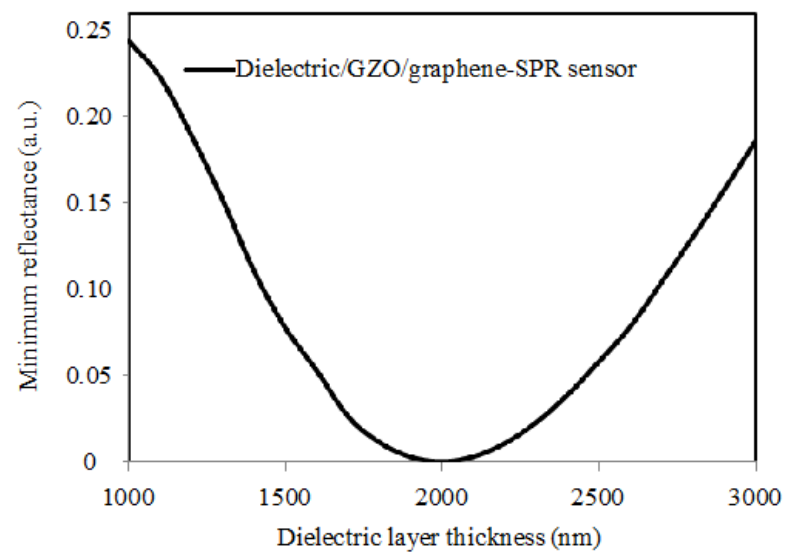

(b)

Fig. 2 Plot of the minimum reflectance as function of (a) GZO thickness and (b) dielectric thickness of the proposed GZO/graphene SPR sensor for the optimization of GZO and dielectric thickness [the thickness used for graphene is $0.34 \mathrm{~nm}$, the dielectric thickness varies from $1000 \mathrm{~nm}$ to $3000 \mathrm{~nm}$, the GZO thickness varies from $12 \mathrm{~nm}$ to $30 \mathrm{~nm}$, and RIs for the dielectric medium and sensing medium are $n_{d}=1.34$ and $n_{s}=1.33$, respectively $]$.

So, the optimized thickness of $20 \mathrm{~nm}$ is chosen for the proposed SPR sensor. To get the optimized dielectric thickness, $R_{\min }$ is calculated for dielectric thickness variation from $1000 \mathrm{~nm}$ to $3000 \mathrm{~nm}$ at $20 \mathrm{~nm}$ optimized thicknesses of GZO as shown in Fig. 2(b). The minimum reflectance is obtained at a dielectric thickness of $2000 \mathrm{~nm}$ for the proposed SPR sensor. So, the optimized dielectric thickness of $2000 \mathrm{~nm}$ and RI of 1.34 are laid between the prism and GZO to get an improved FOM and DA for the proposed SPR sensor [8]. Thereafter, monolayer graphene is laid over GZO to detect biomolecules efficiently due to the larger surface to volume ratio of graphene. The thickness used for monolayer graphene is $0.34 \mathrm{~nm}$ and the complex RI at $1550 \mathrm{~nm}$ operating wavelength is given by [16]

$$
n_{g}=3+\mathrm{i} *\left\{\left(5.446 * 10^{-6}\right) / 3\right\} * \lambda
$$

where $\lambda$ is in $\mu \mathrm{m}$. RI for the sensing medium considered here is $1.33-1.34$. When an aqueous solution containing biomolecules makes contact with the sensor surface, as a result biomolecule present in the sample binds due to pi-interaction between the graphene and biomolecules. This increases RI of the sensor surface on adsorption of biomolecules and leads to shifting dip position of the SPR curve. Reflectance computation for the plotting resonance curve is shown briefly in the next sub-section.

\subsection{Transfer matrix method}

It is well known as an efficient method for calculating reflectance, which does not consider 
approximations [23, 24]. It is based on multilayer modeling. This modeling is used here for the proposed SPR biosensor design to calculate the reflectivity obtained through attenuated total reflection at the $1550 \mathrm{~nm}$ wavelength. The reflectivity obtained is then plotted as a function of the incident angle also known as SPR curves for the proposed design. Thereafter, the performance of the proposed SPR biosensor design is evaluated through the SPR curve. The performance of the proposed biosensor is evaluated in terms of sensitivity, DA, and FOM from the SPR curve of the proposed SPR biosensor. PD and PL of SPs in the proposed design are also calculated to analyze its performance. The respective modeling and definitions of performance parameters evaluated throughout this paper are discussed in detail in Appendix.

\section{Results and discussion}

\subsection{Reflectance curve analysis}

This section describes the reflectance curve obtained for the proposed sensor design by using the transfer matrix method. The SPR sensor design shown in Fig. 1(a) shows the use of GZO in place of metal for the Kretschmann configuration based SPR sensor. The thickness taken for GZO is $50 \mathrm{~nm}$ for the GZO/graphene-based SPR sensor. Reflectance curves are plotted for the GZO/graphene SPR sensor as per the SPR sensor design in Figs. 1(a) and 3 by red dotted lines. The resonance angle and minimum reflectance $\left(R_{\text {min. }}\right)$ obtained for the $\mathrm{GZO} /$ graphene SPR are $83.02^{\circ}$ and 0.0437 a.u., respectively. But the larger spectral width or larger FWHM of $19.99^{\circ}$ gives the poorer DA. DA calculated for the above obtained FWHM value is 0.05 degree $^{-1}$ for the GZO/graphene SPR sensor. As per Sarid et al., the use of a thin metal layer between two dielectric media can give sharper SPR curves. So, to improve the DA of a nanostructured TCO based SPR sensor, a dielectric layer is introduced between the GZO and graphene as shown in Fig. 1(b). The reflectance curve corresponding to Fig. 1(b) is plotted and shown in Fig. 3 for the GZO/graphene SPR sensor with the dielectric by the black solid line. The resonance angle and minimum reflectance $\left(R_{\min }\right)$ obtained for the proposed SPR sensor are $63.0248^{\circ}$ and 0 a.u., respectively. FWHM and DA obtained for the proposed SPR sensor are $0.6^{\circ}$ and 1.67 degree $^{-1}$, respectively.

Figure 3 indicates that the FWHM of the SPR sensor as per the schematic diagram in Fig. 1(a) is much wider in comparison with the FWHM of the SPR sensor as per the sensor design in Fig. 1(b). This shows that the introduction of the dielectric layer between the prism and plasmonic GZO excites SPPs to a higher penetration depth and results in a narrower spectral width. Hence, the improved DA and FOM can be obtained for the GZO/Graphene SPR biosensor with dielectric. To compare DA of the proposed design with that of the conventional SPR sensor utilizing $50 \mathrm{~nm}$ gold layer for plasmon generation, the resonance angle, minimum reflectance $\left(R_{\text {min. }}\right)$, and FWHM are calculated for the conventional SPR sensor. The resonance angle, minimum reflectance $\left(R_{\text {min. }}\right)$, and FWHM obtained for the conventional SPR sensor at 1.33 RI of the sensing medium are $63.751^{\circ}, 0.2406$ a.u., and $0.65^{\circ}$, respectively. Hence, DA for the conventional SPR sensor is 1.54 degree $^{-1}$, which is less than that of the proposed design utilizing GZO for SPPs generation.

The proposed SPR sensor is sensitive to the change in thickness and RI of the dielectric layer. So, the effect of increasing dielectric layer thickness and $\mathrm{RI}$ on the resonance curve is analysed in Figs. 4 and 5 , respectively for the proposed SPR sensor. It is observed from Fig. 4 that the resonance angle, minimum reflectance $\left(R_{\text {min. }}\right)$, FWHM, and DA obtained for dielectric thicknesses of $2000 \mathrm{~nm}$, $2200 \mathrm{~nm}, 2400 \mathrm{~nm}, 2600 \mathrm{~nm}$, and $2800 \mathrm{~nm}$ at sensing layer RI of 1.33 for the GZO/graphene SPR sensor with dielectric are $\left(63.0248^{\circ}, 63.1^{\circ}, 63.12^{\circ}\right.$, $63.14^{\circ}$, and $63.17^{\circ}$ ), (0a.u., 0.01063 a.u., 0.03877 a.u., 
0.0784 a.u., and 0.1304 a.u. $),\left(0.6^{\circ}, 0.53^{\circ}, 0.47^{\circ}\right.$, $0.42^{\circ}$, and $\left.0.36^{\circ}\right)$, and $\left(1.67\right.$ degree $^{-1}, 1.89$ degree $^{-1}$, 2.13 degree $^{-1}, 2.38$ degree $^{-1}$, and 2.78 degree $^{-1}$ ), respectively. The resonance angle shifts to the higher value and the spectral width of SPR curves becomes narrower with increasing the dielectric layer thickness as observed in Fig. 4. This clearly indicates that there is an improvement in DA due to the narrower FWHM for the higher dielectric layer thickness.

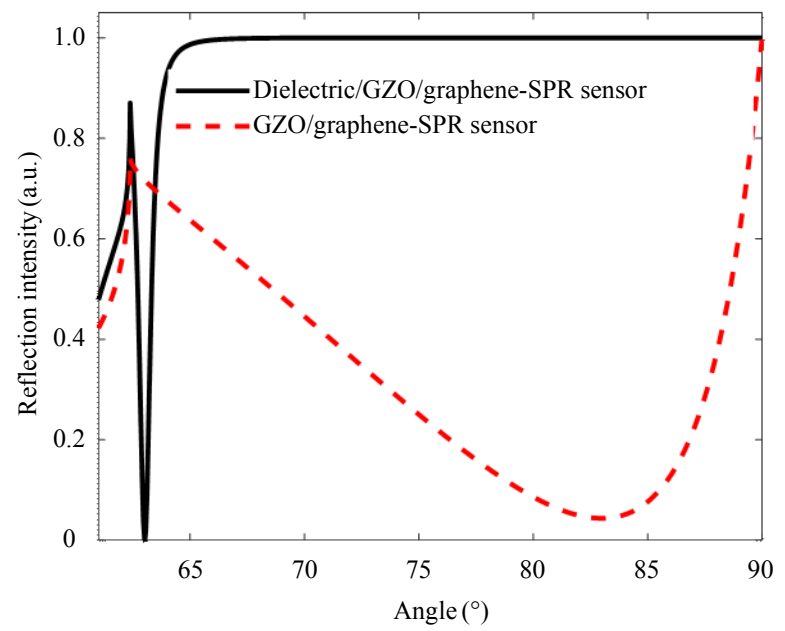

Fig. 3 Reflectance curve for the GZO/graphene SPR sensor with and without using dielectric (thicknesses used for graphene, dielectric, and GZO are $0.34 \mathrm{~nm}, 2000 \mathrm{~nm}$, and $20 \mathrm{~nm}$, respectively; RIs for the dielectric medium and sensing medium are $n_{d}=1.34$ and $n_{s}=1.33$, respectively).

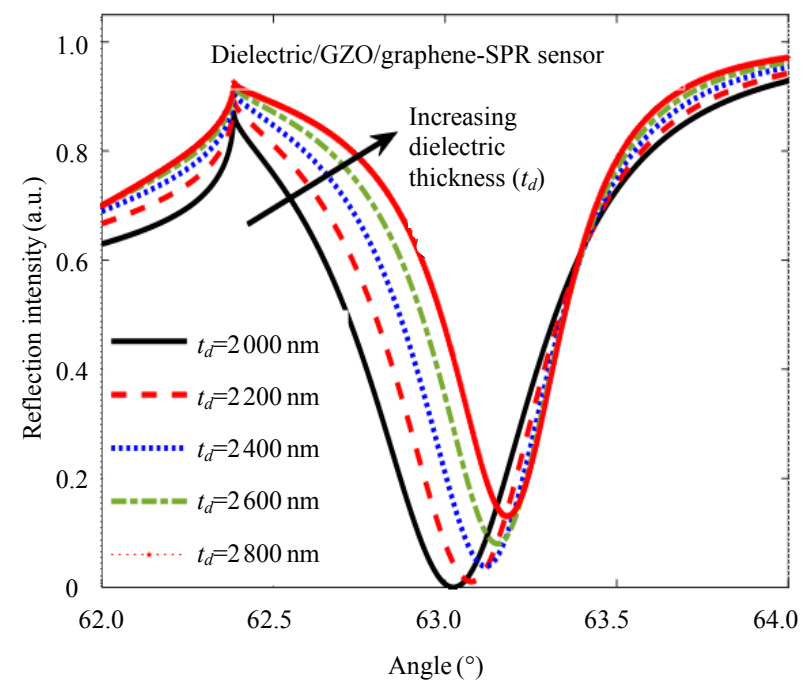

Fig. 4 Reflectance curve of the proposed SPR sensor for dielectric thickness variation from $2000 \mathrm{~nm}$ to $2800 \mathrm{~nm}$ (thicknesses used for graphene and GZO are $0.34 \mathrm{~nm}$ and $20 \mathrm{~nm}$, respectively; RIs for the dielectric medium and sensing medium are $n_{d}=1.34$ and $n_{s}=1.33$, respectively).
To check the effect of increasing dielectric RI on the proposed design, Fig. 5 is plotted. Figure 5 shows that the resonance angle, minimum reflectance $\left(R_{\text {min. }}\right)$, FWHM, and DA for the GZO/graphene SPR sensor with dielectric are $\left(63.0248^{\circ}, 63.46^{\circ}, 63.97^{\circ}\right.$, $64.54^{\circ}$, and $\left.65.18^{\circ}\right),(0$ a.u., 0.0218 a.u, 0.0748 a.u., 0.1427 a.u., and 0.2149 a.u. $),\left(0.6^{\circ}, 0.78^{\circ}, 0.93^{\circ}\right.$, $1.03^{\circ}$, and $\left.1.09^{\circ}\right)$, and $\left(1.67\right.$ degree $^{-1}, 1.28$ degree $^{-1}$, 1.075 degree $^{-1}, 0.971$ degree $^{-1}$, and 0.917 degree $^{-1}$ ), respectively. It is observed from Fig. 5 that the resonance angle shifts to a higher value and the spectral width of curves becomes broader with increasing dielectric layer RI. To get the higher DA and FOM for the proposed SPR biosensor, we have kept the lower value of dielectric layer RI i.e., 1.34.

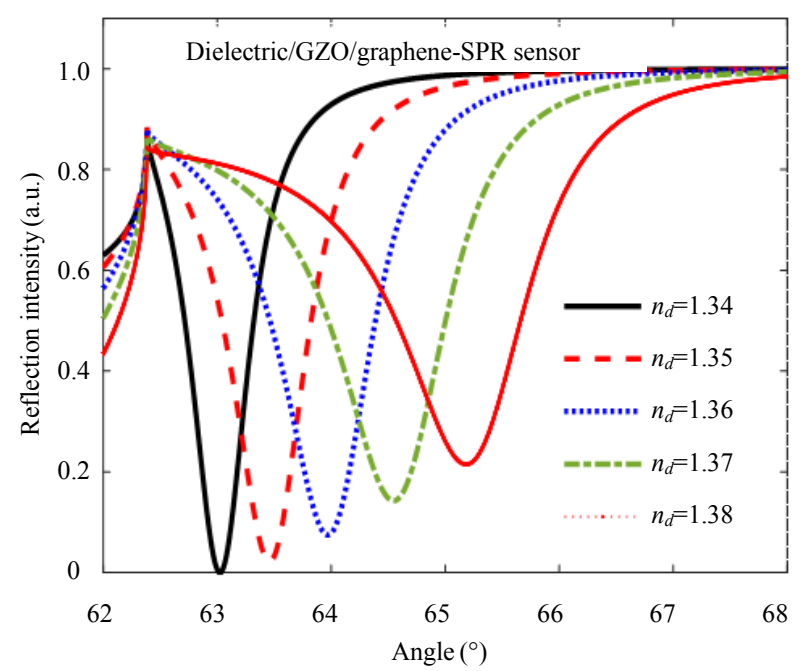

Fig. 5 Reflectance curve for the proposed SPR sensor for dielectric RI variation from 1.34 to 1.38 (thicknesses used for graphene, dielectric, and GZO are $0.34 \mathrm{~nm}, 2000 \mathrm{~nm}$, and $20 \mathrm{~nm}$, respectively; RI for the sensing medium is $n_{s}=1.33$ ).

The effect of an increasing number of graphene layers for the GZO/graphene SPR sensor with dielectric is observed in Fig. 6. It indicates that the higher resonance angle shifts and the broader spectral width of curves are obtained with the increasing number of the graphene layer. FWHM increases for a higher number of graphene layers but DA reduces due to the damping effect of SPs for a higher number of graphene layers. So for the accurate detection of biomolecules, we have utilized 
monolayer graphene for the proposed SPR biosensor.

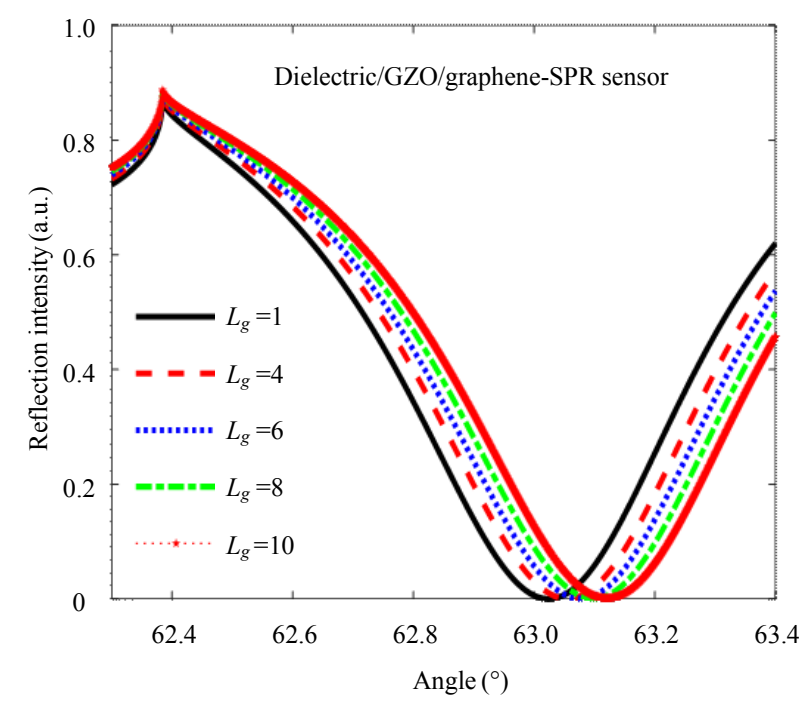

Fig. 6 Reflectance curves for proposed SPR sensor for 1 layer - 10 layers of graphene (thicknesses used for graphene, dielectric, and GZO are $0.34 \mathrm{~nm}, 2000 \mathrm{~nm}$, and $20 \mathrm{~nm}$, respectively; RIs for the dielectric medium and sensing medium are $n_{d}=1.34$ and $n_{s}=1.33$, respectively).

\subsection{Performance analysis of the proposed SPR biosensor}

This subsection analyses the performance of the proposed GZO/Graphene SPR sensor with a dielectric in terms of sensitivity, DA, and FOM, which analyses the variation in sensitivity and DA for dielectric thickness variation from $1500 \mathrm{~nm}-$ $3000 \mathrm{~nm}$ at 1.34 sensing layer RI for the proposed SPR sensor. An increase in the dielectric layer thickness from $1500 \mathrm{~nm}$ to $3000 \mathrm{~nm}$ shows a decrement in sensitivity from $61 \%$ RIU to $44^{\circ} /$ RIU for the proposed SPR sensor.

Figure 7(a) analyses the variation in sensitivity and DA for dielectric thickness variation from $1500 \mathrm{~nm}$ to $3000 \mathrm{~nm}$ at 1.34 sensing layer RI for the proposed SPR sensor. An increase in dielectric layer thickness from $1500 \mathrm{~nm}$ to $3000 \mathrm{~nm}$ shows a decrease in sensitivity from $61 \%$ RIU to $44^{\circ}$ /RIU for the proposed SPR sensor. As observed in Fig. 7(a), DA increases from 1.82 degree $^{-1}$ to 8.33 degree $^{-1}$ with an increment in dielectric layer thickness $(1500 \mathrm{~nm}$ - $3000 \mathrm{~nm}$ ) for the proposed SPR sensor. The variation in FOM with respect to dielectric layer thickness variation for the proposed SPR sensor is shown in Fig. 7(b). FOM increases with an increase in dielectric layer thickness for the proposed SPR sensor. Figure 7(b) indicates that FOM increases for dielectric thickness variation of $1500 \mathrm{~nm}-3000 \mathrm{~nm}$ from $110.9 \mathrm{RIU}^{-1}$ to $366.7 \mathrm{RIU}^{-1}$ for the proposed GZO/graphene SPR sensor. The highest FOM of $366.7 \mathrm{RIU}^{-1}$ is observed for the proposed SPR sensor at $3000 \mathrm{~nm}$ dielectric layer thickness.

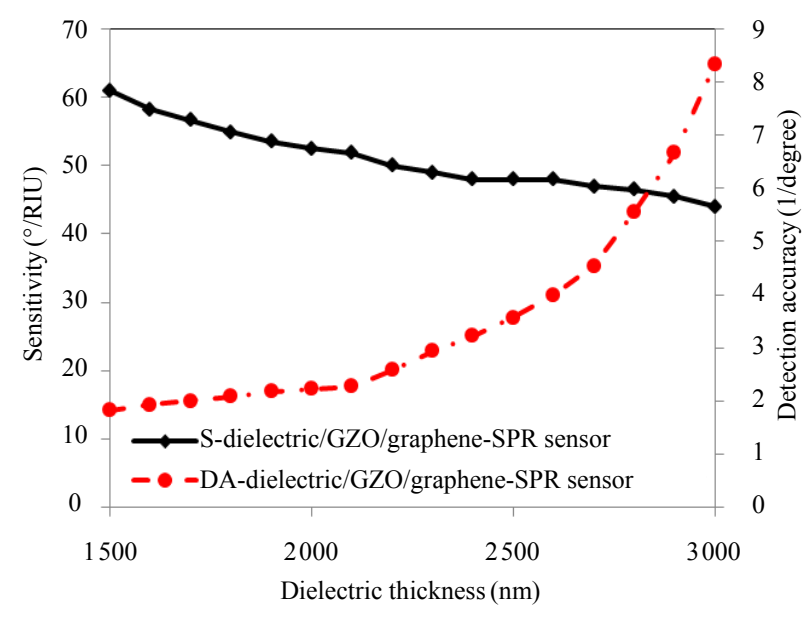

(a)

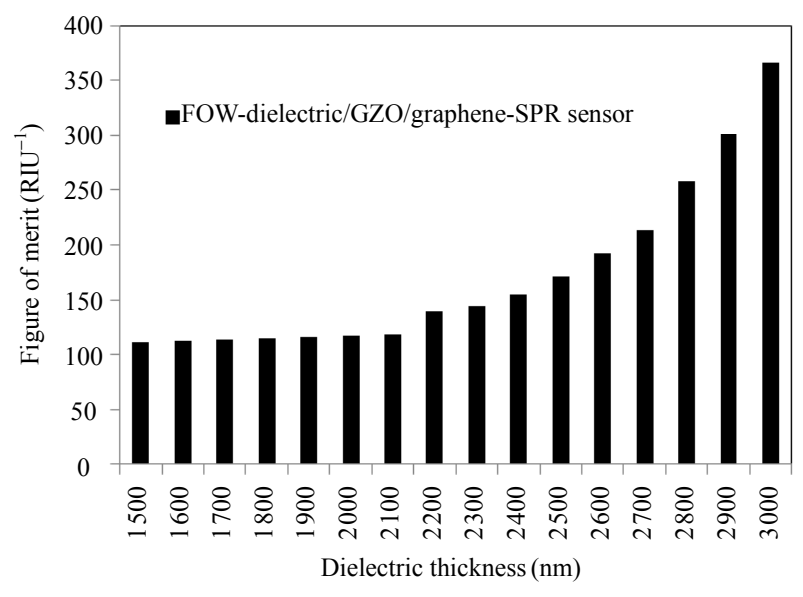

(b)

Fig. 7 Plot of (a) sensitivity and DA vs. dielectric thickness and (b) FOM vs. dielectric thickness for dielectric layer thickness variation of $1500 \mathrm{~nm}-3000 \mathrm{~nm}$ for the proposed SPR sensor (thicknesses used for graphene and GZO are $0.34 \mathrm{~nm}$ and $20 \mathrm{~nm}$, respectively; RIs for the sensing medium and dielectric medium are $n_{s}=1.33$ and $n_{d}=1.34$, respectively). 
Now, a variation of sensitivity, DA, and FOM as a function of RI of the sensing layer $(1.33-1.34)$ are plotted for the proposed SPR sensor in Figs. 8(a) and 8 (b) at $2000 \mathrm{~nm}$ dielectric layer thickness. Both sensitivity and DA increase with increasing RI of the sensing layer from 1.33 to 1.34 . Sensitivity increases from $44 \%$ RIU to $52 \%$ RIU for the proposed SPR sensor as shown in Fig. 8(a). Similarly, DA increases from 1.64 degree $^{-1}$ to 2.33 degree $^{-1}$ for the proposed

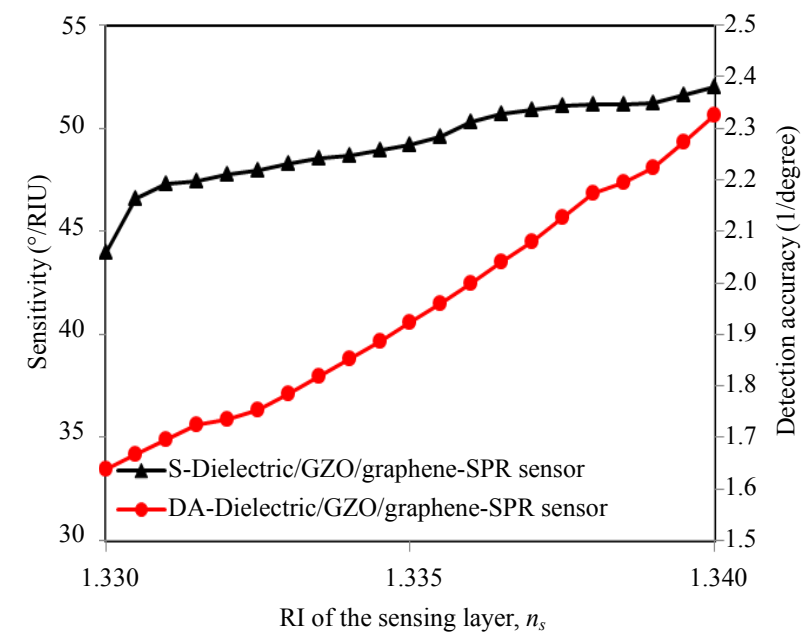

(a)
SPR sensor as shown in Fig. 8(a). Almost a two-fold increment in sensitivity is achieved for the proposed GZO based SPR biosensor in comparison with [8]. Now Fig. 8(b) indicates that FOM increases form $77 \mathrm{RIU}^{-1}$ to $120.97 \mathrm{RIU}^{-1}$ for the proposed SPR sensor. This work clearly demonstrates that the higher FOM is achieved when GZO is used in place of metal as a plasmonic material for the proposed SPR biosensor.

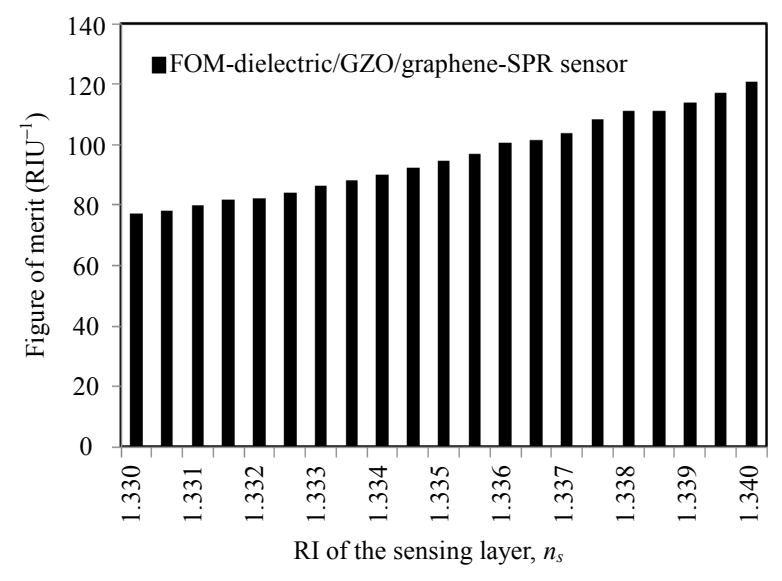

(b)

Fig. 8 Plot of (a) sensitivity and DA for the proposed SPR sensor and (b) FOM for GZO/graphene LRSPR sensor as a function of sensing layer RI (1.33 - 1.34) (thicknesses used for graphene, dielectric layer, and GZO are $0.34 \mathrm{~nm}, 2000 \mathrm{~nm}$, and $20 \mathrm{~nm}$, respectively; RIs for the sensing medium and dielectric medium are $n_{s}=1.33-1.34$ and $n_{d}=1.34$, respectively).

PD of SPs in the transverse direction and PL of SPs along the interface are important parameters to evaluate the performance of the SPR sensor. Figures 9(a) - 9(c) show the TM field distribution of the conventional SPR sensor, GZO/graphene SPR, and GZO/graphene SPR with dielectric, respectively.

TM field plot for the GZO/graphene SPR sensor is shown in Fig. 9(b). It indicates that the field is almost constant in GZO nanostructured TCO becomes the maximum at the $\mathrm{GZO} /$ graphene interface and after that it decays exponentially away from the interface. Figure 9(c) shows the propagation of the TM field at different interfaces of the dielectric/GZO/graphene/sensing medium for the proposed SPR biosensor.

Figure 9(a) indicates the TM field plot for the conventional SPR sensor with respect to the distance normal to an interface which is exactly as explained in the literature. It indicates that the intensity of the TM field increases when the metal thickness becomes maximum at the metal/sensing medium interface, and then it decreases exponentially away from the interface in the sensing medium [24].

The plot shows that the field is guided in the dielectric medium, but at the GZO/dielectric interface, the field becomes almost constant inside GZO with SPs generation. Two subplots are plotted here to give a clear picture of TM field variation at different interfaces. The subplot at the top indicates that the field is almost constant within GZO i.e., plasmonic layer for thickness variation from $2000 \mathrm{~nm}$ to $2020 \mathrm{~nm}$. The second subplot indicates that the TM field becomes maximum at the $\mathrm{GZO/graphene} \mathrm{interface} \mathrm{due} \mathrm{to} \mathrm{the} \mathrm{strong} \mathrm{and} \mathrm{stable}$ 
attachment of biomolecules on graphene, and then it exponentially decreases in the sensing medium.

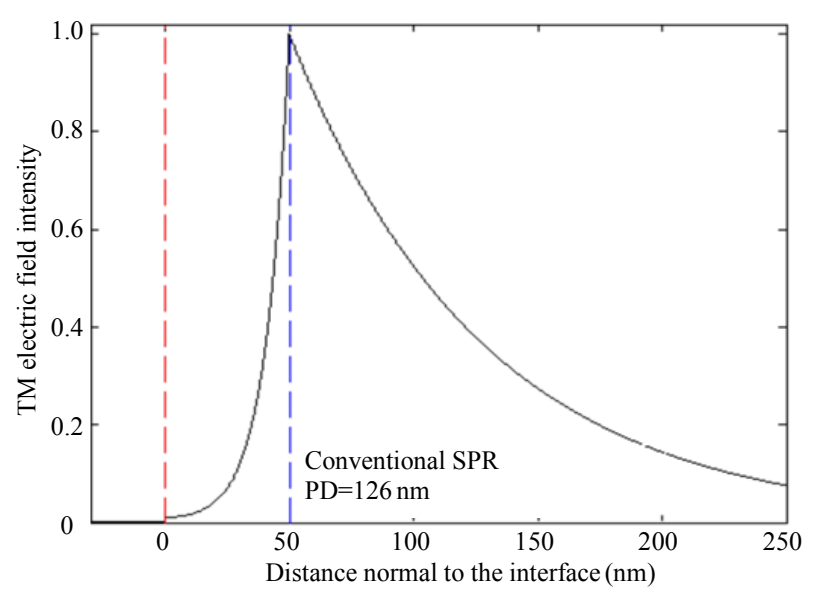

(a)

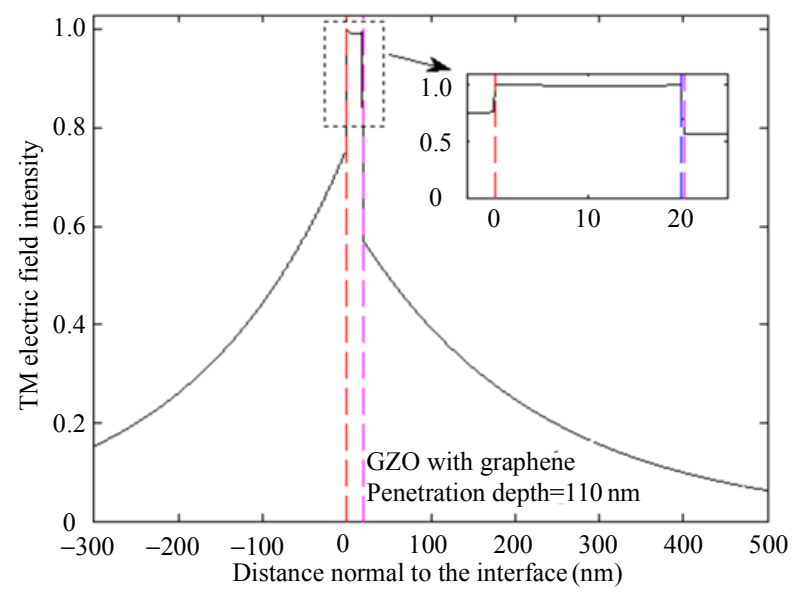

(b)

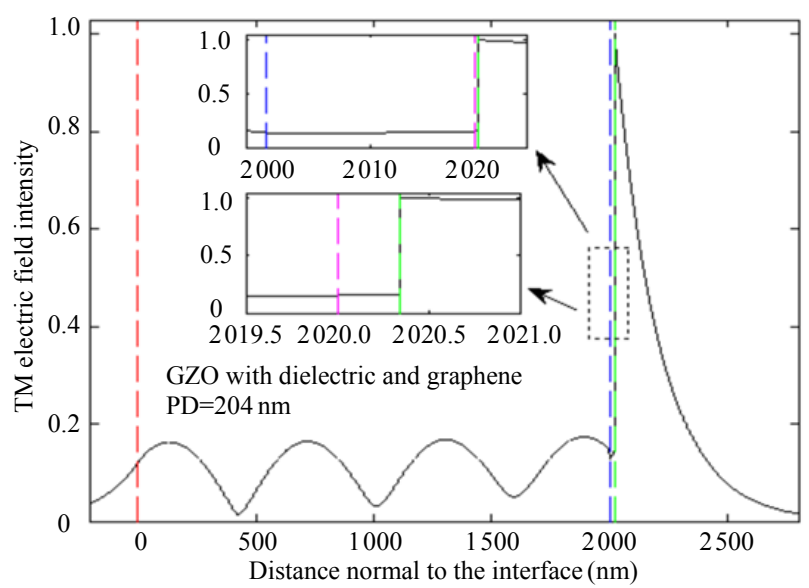

(c)

Fig. 9 Plot of TM field distribution vs. distance normal to the interface (nm) for (a) conventional SPR sensor, (b) GZO/ graphene SPR sensor, and (c) dielectric/GZO/graphene SPR sensor.

PDs are calculated here for all three cases in
Figs. 9(a) -9 (c) to analyse the depths of the TM field in sensing medium and compare their performances. PDs observed from Figs. 9(a) - 9(c) for the conventional SPR sensor, GZO/graphene SPR, and GZO/graphene SPR with dielectric are $126 \mathrm{~nm}, 110 \mathrm{~nm}$, and $204 \mathrm{~nm}$, respectively. It is greater for the conventional SPR than that of GZO/graphene SPR as SPs excited through nanostructured TCOs thin films results in a broader resonance curve. This was also experimentally verified by Kim et al. [9]. The highest PD of $204 \mathrm{~nm}$ is observed for the GZO/graphene SPR sensor with dielectric (the proposed SPR sensor), indicating a larger interaction volume of the field in the sensing medium which makes it suitable for the detection of larger size biomolecules like secretary vesicles and lysosomes.

The propagation lengths of propagating SPs in the lateral direction are also calculated here for the conventional SPR sensor, GZO/graphene SPR, and $\mathrm{GZO/graphene} \mathrm{SPR} \mathrm{with} \mathrm{dielectric.} \mathrm{PLs} \mathrm{obtained} \mathrm{for}$ the conventional SPR sensor GZO/graphene SPR and $\mathrm{GZO} /$ graphene SPR with dielectric are $0.52 \mu \mathrm{m}$, $0.67 \mu \mathrm{m}$, and $0.69 \mu \mathrm{m}$, respectively.

Table 2 compares PD $(\mathrm{nm})$ and PL $(\mu \mathrm{m})$ of the electromagnetic field for the conventional SPR sensor, GZO/graphene SPR, and GZO/graphene SPR with dielectric. The highest PD and PL are achieved for the proposed SPR biosensor. Thus, the proposed SPR sensor is suitable for the detection of larger size biomolecules at the telecommunication wavelength.

Table 2 PL and PD calculated for the conventional SPR sensor, GZO/graphene SPR, and GZO/graphene SPR with dielectric.

\begin{tabular}{cccc}
\hline No. & Sensor type & PL $(\mu \mathrm{m})$ & PD (nm) \\
\hline 1 & $\begin{array}{c}\text { Conventional SPR sensor } \\
(\text { prism/Au/sensing medium) }\end{array}$ & 0.52 & 126 \\
\hline 2 & $\begin{array}{c}\text { GZO/graphene SPR sensor } \\
(\text { prism/GZO/sensing medium) }\end{array}$ & 0.67 & 110 \\
\hline 3 & $\begin{array}{c}\text { Proposed SPR biosensor } \\
(\text { prism/dielectric/ GZO/sensing } \\
\text { medium) }\end{array}$ & 0.69 & 204 \\
\hline
\end{tabular}




\section{Conclusions}

We propose an SPR biosensor that utilizes nanostructured transparent conducting oxide (GZO) instead of using conventional metals like gold and silver for SPPs generation. Graphene is used as BRE for better binding of biomolecules. The higher penetration depth of SPs in the sensing region leads to the detection of larger size biomolecules. Total attenuated reflection geometry is used to perform simulations analytically for the Kretschmann configuration based proposed SPR biosensor. The NIR wavelength of $1550 \mathrm{~nm}$ has been chosen to avoid photo-damage to biosamples. Sensitivity, DA, and FOM are calculated for the proposed SPR biosensor. The highest FOM of $366.7 \mathrm{RIU}^{-1}$ is achieved for the proposed SPR biosensor at $3000 \mathrm{~nm}$ dielectric thickness. PD and PL are also calculated to compare with the performance of the conventional SPR sensor, GZO/graphene SPR, and proposed SPR biosensor i.e., GZO/graphene SPR with dielectric. Advancement in fabrication techniques leads to realizing the proposed SPR sensor practically in future due to the fabrication possibilities of nanostructured GZO and graphene. We believe that our work will open new possibilities of using nanostructured TCO (GZO) as SPs generating material in plasmonic sensing for its application in drug diagnostic, food safety, and biological detection.

\section{Appendix: transfer matrix method}

This method is used here to calculate reflectivity for the proposed SPR design which is arranged in multi-layered fashion. Here, layers with thicknesses $d_{k}$, dielectric constant $\varepsilon_{k}$, and refractive index $n_{k}$ are arranged along the $z$-axis. The medium is assumed to be nonmagnetic, isotropic, and uniform. As per the boundary condition, the tangential fields at $Z=Z_{1}=0$ can be obtained in terms of the tangential field at $Z=Z_{N-1}$ on applying boundary conditions:

$$
\left[\begin{array}{l}
U_{1} \\
V_{1}
\end{array}\right]=M_{i j}\left[\begin{array}{l}
U_{N-1} \\
V_{N-1}
\end{array}\right]
$$

where $\left[U_{1}, V_{1}\right]$ signifies the tangential fields of electric and magnetic fields at the boundary of the first and $\left[U_{N-1}, V_{N-1}\right]$ represents the tangential fields of electric and magnetic fields at the boundary of the $N$ th layer. The characteristic transfer matrix $\left(M_{i j}\right)$ for the stacked multilayer structure of the proposed sensor design, for TM-polarized light, is as shown below:

$$
M_{i j}=\left(\prod_{k=2}^{n-1} M_{k}\right)_{i j}=\left[\begin{array}{ll}
M_{11} & M_{12} \\
M_{21} & M_{22}
\end{array}\right]
$$

with

$$
M_{k}=\left[\begin{array}{cc}
\cos \beta_{k} & \left(-\mathrm{i} \sin \beta_{k}\right) / q_{k} \\
-\mathrm{i} q_{k} \sin \beta_{k} & \cos \beta_{k}
\end{array}\right]
$$

where

$$
q_{k}=\left(\frac{\mu_{k}}{\varepsilon_{k}}\right)^{1 / 2} \cos \theta_{k}=\frac{\left(\varepsilon_{k}-n_{1}^{2} \sin ^{2} \theta_{1}\right)^{1 / 2}}{\varepsilon_{k}}
$$

and

$$
\beta_{k}=\frac{2 \pi}{\lambda}\left(\varepsilon_{k}-n_{1}^{2} \sin ^{2} \theta_{1}\right)^{1 / 2}
$$

where $n_{1}, \theta_{1}$, and $\lambda$ represent the refractive index of the prism, incident angle, wavelength of incident light at the base of prism respectively; $\theta_{k}, \varepsilon_{k}$, and $\mu_{k}$ are the incident angle, dielctric constant, and permeability, respectively of the $k$ th layer. The reflection coefficient obtained for TM-polarized light is given below:

$$
r_{p}=\frac{\left(M_{11}+M_{12} q_{N}\right) q_{1}-\left(M_{21}+M_{22} q_{N}\right)}{\left(M_{11}+M_{12} q_{N}\right) q_{1}+\left(M_{21}+M_{22} q_{N}\right)}
$$

where $q_{1}$ and $q_{N}$ are relative components of the first and $N$ th layer, respectively and can be calculated from (4). The reflectance $\left(R_{p}\right)$ for the multilayer configuration is given as

$$
R_{p}=\left|r_{p}\right|^{2} .
$$

Now, the reflectance obtained may be plotted as a function of the incident angle known as SPR curves for the proposed design. The performance of the proposed biosensor is evaluated in terms of sensitivity, detection accuracy, FOM, PD, and PL of SPs in the proposed design. Definitions of 
performance parameters are given as below:

(1) Sensitivity $(S)$ is the ratio of resonance angle shift $\left(\partial \theta_{\text {res }}\right)$ to shift in RI of the sensing layer $\left(\partial n_{s}\right)$ which can be calculated from SPR curves and its measuring unit is ${ }^{\circ} / \mathrm{RIU}$. It may be represented as

$$
S=\frac{\partial \theta_{\text {Res }}}{\partial n_{s}}(\% / \text { RIU }) \text {. }
$$

(2) $D A$ is the reciprocal of FWHM and its unit is degree $^{-1}$.

$$
D A=\frac{1}{F W H M}\left(\text { degree }^{-1}\right)
$$

where $F W H M$ is the spectral width of the SPR curve at which $50 \%$ reflectance is obtained.

(3) FOM may be defined as a product of sensitivity and $D A$ of the SPR biosensor and its unit is RIU $^{-1}$.

$$
F O M=S * D A=\frac{\partial \theta_{\mathrm{Res}}}{\partial n_{s}} * \frac{1}{F W H M}\left(\mathrm{RIU}^{-1}\right) .
$$

(4) $P D$ is defined as the distance travelled by the electromagnetic field normal to the GZO and dielectric interface in the sensing medium where the amplitude of the field decays to $1 / \mathrm{e}$ or $37 \%$ from its maximum value [4]. $P D$ of SPs in GZO and dielectric can be analytically approximated as

$$
\partial_{\mathrm{GZO}}=\frac{1}{\left|k_{z}\right|} \approx\left|\frac{\lambda}{2 \pi} \sqrt{\left(\frac{\varepsilon_{\mathrm{GZO}}^{\prime}+\varepsilon_{d}}{\left(\varepsilon_{\mathrm{GZO}}^{\prime}\right)^{2}}\right)}\right|
$$

and

$$
\partial_{\text {dielectric }}=\frac{1}{2 k_{x}^{\prime \prime}} \approx\left|\frac{\lambda}{2 \pi} \sqrt{\left(\frac{\varepsilon_{\mathrm{GZO}}^{\prime}+\varepsilon_{d}}{\left(\varepsilon_{d}\right)^{2}}\right)}\right|
$$

where $\lambda$ is the operating wavelength, and $\varepsilon_{\text {GZO }}^{\prime}$ and $\varepsilon_{d}$ are the real part of relative permittivity of GZO and relative permittivity of dielectric, respectively. Here, $k_{x}$ and $k_{z}$ are the wave vectors of surface plasmons in the $x$ and $z$ directions respectively; $k_{x}$ " is imaginary part of wave vector of surface plasmons in the $x$ direction.

(5) $P L$ is defined as the distance traveled by SPs along the direction of propagation at which its intensity decreases by factor of $1 / \mathrm{e}$ from its initial value [4]. It may be expressed as inverse of twice of imaginary part of complex propagation constant of
SPs in the lateral direction and given as

$$
L=\frac{1}{2 k_{x}^{\prime \prime}} \approx \frac{c}{\omega} \frac{\left(\varepsilon_{\mathrm{GZO}}^{\prime}\right)^{2}}{\varepsilon_{\mathrm{GZO}}^{\prime \prime}}\left(\frac{\varepsilon_{\mathrm{GZO}}^{\prime}+\varepsilon_{d}}{\varepsilon_{\mathrm{GZO}}^{\prime} \varepsilon_{d}}\right)^{3 / 2} .
$$

where $\omega, c$, and $\varepsilon_{\mathrm{GZO}}^{\prime \prime}$ are the incident light frequency, velocity of light in vacuum, and imaginary part of dielectric constant of GZO, respectively.

\section{Acknowledgment}

This work is partially supported by the Board of Research in Nuclear Sciences (BRNS) (Grant No. 34/14/10/2017-BRNS/34285), Department of Atomic Energy (DAE), and Government of India.

Open Access This article is distributed under the terms of the Creative Commons Attribution 4.0 International License (http://creativecommons.org/licenses/by/4.0/), which permits unrestricted use, distribution, and reproduction in any medium, provided you give appropriate credit to the original author(s) and the source, provide a link to the Creative Commons license, and indicate if changes were made.

\section{References}

[1] J. B. Maurya, Y. K. Prajapati, S. Raikwar, and J. P. Saini, "A silicon-black phosphorous based surface plasmon resonance sensor for the detection of $\mathrm{NO}_{2}$ gas," Optik, 2018, 160: 428-433.

[2] J. Hamola, "Surface plasmon resonance sensors for detection of chemical and biological species," Chemical Reviews, 2008, 108(2): 462-493.

[3] S. Pal, A. Verma, J. P. Saini, and Y. K. Prajapati, "Sensitivity enhancement using silicon-black phosphorus-TDMC coated surface plasmon resonance biosensor," IET Optoelectronics, 2019, 13(4): 196-201.

[4] J. Homola, Surface plasmon resonance based sensors. Springer series on chemical sensors and biosensors, vol. 4. Berlin: Springer-Verlag Berlin Heidelberg, 2006: 45-67.

[5] S. Pal, A. Verma, Y. K. Prajapati, and J. P. Saini, "Influence of black phosphorous on performance of surface plasmon resonance biosensor," Optical and Quantum Electronics, 2017, 49: 403.

[6] P. R. West, S. Ishii, G. V. Naik, N. K. Emani, V. M. Shalaev, and A. Boltasseva, "Searching for better plasmonic materials", Laser \& Photonics Reviews, 2010, 4(6): 795-808.

[7] A. K Pandey, A. K. Sharma, and R. Basu, "Fluoride glass-based surface plasmon sensor in infrared 
region: performance evaluation," Journal of Physics D: Applied Physics, 2017, 50(18): 185103-1-185103-10.

[8] B. Ruan, Q. You, J. Zhu, L. Wu, J. Guo, X. Dai, et al., "Improving the performance of an SPR biosensor using long-range surface plasmon of Ga-doped zinc oxide," Sensors, 2018, 18(7): 2098.

[9] J. Kim, G. V. Naik, N. K. Emami, U. Guler, and A. Boltasseva, "Plasmonic resonances in nanostructured transparent conducting oxides films," IEEE Journal of Selected Topics in Quantum Electronics, 2013, 19(3): 4601907.

[10] G. V. Naik, J. Kim, and A. Boltasseva, "Oxides and nitrides as alternative plasmonic materials in the optical range," Optical Materials Express, 2011, 1(6): 1090-1099.

[11] R. Slavik and J. Homola, "Ultrahigh resolution long range surface plasmon-based sensor," Sensors and Actuators B: Chemical, 2007, 123(1): 10-12.

[12] A. W. Wark, H. J. Lee, and R. M. Corn, "Long-range surface plasmon resonance imaging for bioaffinity sensors," Analytical Chemistry, 2005, 77(13): 3904-3907.

[13] D. Sarid, "Long-range surface-plasma waves on very thin metal films," Physical Review Letters, 1981, 47(26): 1927-1930.

[14] B. Song, D. Li, W. P. Qi, M. Elstner, C. H. Fan, and H. P. Fang, "Graphene on Au (111): a highly conductive material with excellent absorption properties for high-solution bio/nanodetection and identification," ChemPhysChem, 2010, 11(3): 585-589.

[15] S. Pal, A. Verma, S. Raikwar, and J. P. Saini, "Detection of DNA hybridization using graphene-coated black phosphorus surface plasmon resonance sensor," Applied Physics A, 2018, 124(5): 394.

[16]A. Verma, A. Prakash, and R. Tripathi, "Comparative study of surface plasmon resonance biosensor based on metamaterial and graphene," Silicon, 2017, 9(3):
309-320.

[17] L. Wu, J. Guo, Q. Wang, S. Lu, X. Dai, Y. Xiang, et al., "Sensitivity enhancement by using few-layer black phosphorus-graphene/TMDCs heterostructure in surface plasmon resonance biochemical sensor," Sensors and Actuators B: Chemical, 2017, 249: 542-548.

[18] W. C. Tan, M. Hofmann, Y. Hsieh, M. L. Lu, and Y. F. Chen, "A graphene-based surface plasmon sensor," Nano Research, 2012, 5(10): 695-702.

[19] X. Zhao, T. Huang, P. S. Ping, X. Wu, P. Huang, J. Pan, et al., "Sensitivity enhancement in surface plasmon resonance biochemical sensor based on transition metal dichalcogenides/graphene heterostructure," Sensors, 2018, 18(7): 2056.

[20] S. Chen and C. Lin, "Sensitivity comparison of graphene-based surface plasmon resonance biosensor with $\mathrm{Au}, \mathrm{Ag}$ and $\mathrm{Cu}$ in the visible region," Materials Research Express, 2019, 6(5): 056503-1-056503-8.

[21] L. Liu, M. Wang, L. Jiao, T. Wu, F. Xia, M. Liu, et al., "Sensitivity enhancement of a graphene-barium titanate-based surface plasmon resonance biosensor with an $\mathrm{Ag}-\mathrm{Au}$ bimetallic structure in the visible region," Journal of the Optical Society of America B, 2019, 36(4): 1108-1116.

[22] M. B. Hossain, T. Tasnim, L. F. Abdulrazak, M. M. Rana, and M. R. Islam, "A numerical approach to design the kretschmann configuration based refractive index graphene- $\mathrm{MoS}_{2}$ hybrid layers with $\mathrm{TiO}_{2}-\mathrm{SiO}_{2}$ nano for formalin detection," Photonic Sensors, 2020, 10(2): 134-146.

[23] Y. K. Prajapati, S. Pal, and J. P. Saini, "Effect of metamaterial and silicon layers on performance of surface plasmon resonance biosensor in infrared range," Silicon, 10(4): 1451-1460.

[24] J. B. Maurya, Y. K. Prajapati, and R. Tripathi, "Effect of molybdenum disulfide layer on surface plasmon resonance biosensor for the detection of bacteria," Silicon, 2018, 10(2): 245-256. 